\title{
Physiotherapy managers' views of musculoskeletal physiotherapy service provision in Ireland: a qualitative study
}

\author{
Helen P. French ${ }^{1}$ and Rose Galvin ${ }^{2}$ \\ ${ }^{1}$ School of Physiotherapy, Royal College of Surgeons in Ireland, Dublin, Republic of Ireland \\ ${ }^{2}$ Discipline of Physiotherapy, Department of Clinical Therapies, University of Limerick, Limerick, Republic of Ireland
}

\begin{abstract}
Aim: Integrated multidisciplinary primary healthcare is still in a relatively early stage of development in Ireland, with significant restructuring occurring in the past decade. Musculoskeletal physiotherapy services traditionally provided in acute hospital settings have been relocated into the primary care setting where the physiotherapist works as part of the multidisciplinary team. This study aimed to explore physiotherapy managers' experiences of managing musculoskeletal physiotherapy services in primary care to gain an insight into the opportunities and challenges in service delivery, changing roles and ongoing professional development needs of staff. Participants: Qualitative design using semi-structured interviews with primary care physiotherapy managers in the Republic of Ireland was employed. Results: Five interviews took in a mix of rural and urban areas nationally. The relationship with the GP was an important one in musculoskeletal physiotherapy services in primary care. Physiotherapists were well skilled but opportunities for professional and career development were restricted. Methods of optimising resources in the face of staffing restrictions were identified. Whilst there were many examples of innovations in service delivery, various barriers negatively impacted on optimal service including resource constraints and national strategy. Conclusions: A number of factors that impact on musculoskeletal service delivery in primary care from the perspective of physiotherapy managers were identified in this study. Future research should explore the views of other stakeholders to provide a more thorough understanding of the relevant issues affecting musculoskeletal physiotherapy service provision in primary care in Ireland.
\end{abstract}

Key words: physiotherapy, multidisciplinary teams, primary health care, qualitative research, service provision

Received 23 September 2016; revised 6 June 2017; accepted 1 July 2017;

first published online 14 August 2017

\section{Introduction}

Over the past few decades, health system transformations have led to the development of new models of health service delivery in many countries (Desmeules et al., 2012). Historically, health services in Ireland have evolved from a system which has been fragmented, overly hospital-

Correspondence to: Dr Helen French, Senior Lecturer in Physiotherapy, School of Physiotherapy, Royal College of Surgeons in Ireland, 123 St. Stephens Green, Dublin 2, Republic of Ireland. Email: hfrench@rcsi.ie centric, and focussed on delivering episodes of care, rather than an integrated and continuous model of care (Department of Health, 2016). Primary care predominantly entailed GP care, without the support of a multidisciplinary team (MDT).

In the last decade, there has been a significant shift in healthcare provision due to the launch of the Primary Care Strategy that aimed to transfer $90 \%$ of health services into the community, via primary care teams (PCTs) which include GPs, practice nurses, community nurses, physiotherapists, occupational therapists, speech and language therapists, social 
workers, and home care services who provide a single point of contact into the Irish health system.

Musculoskeletal complaints are one of the most common reasons for seeking primary care and represent a significant economic and social burden to the healthcare system and the general public (Jordan et al., 2010; Bornhoft et al., 2015). Between $20-26 \%$ of the general population seek healthcare for musculoskeletal complaints over the course of a year (Jordan et al., 2010; MacKay et al., 2010) and $14 \%$ of all primary care consultations are by patients with musculoskeletal problems (Jordan et al., 2010). The change in mode of health service delivery in Ireland has resulted in the transfer of musculoskeletal physiotherapy services traditionally provided in acute hospital physiotherapy departments into primary care. Physiotherapists now play an active role in the assessment and management of musculoskeletal complaints in primary care (Desmeules et al., 2012), moving away from the role of the primary care physiotherapist previously in a domiciliary setting managing a varied caseload to being clinic-based managing a high proportion of musculoskeletal conditions. Evidence to date indicates that physiotherapy treatment of musculoskeletal conditions in primary care results in significant health benefits and cost savings (Hay et al., 2006; Nordeman et al., 2006; Hill et al., 2011).

Delivering measurable and sustainable health service delivery improvements is a key metric for healthcare policy makers and clinicians (Roberts, 2013). Evaluation of these care models to date has broadly focussed on quantitative metrics of the process and outcomes of care including waiting times, recovery times and economic evaluations of the service (Desmeules et al., 2012) but qualitative analysis can provide a deeper understanding from the perspective of key stakeholders. We recently explored physiotherapists' experiences of providing musculoskeletal physiotherapy in an Irish primary care setting using a qualitative methodology, to gain an insight into the current facilitators and barriers in service delivery and on-going professional development needs (French and Galvin, 2017). Four major themes emerged from these focus groups including the value of team working, the evolving role of the physiotherapist in primary care, environmental contexts (including physical infrastructure and interaction with acute sites) and factors associated with engagement in continuous professional development (CPD). Following on from an exploration of

Primary Health Care Research \& Development 2018; 19: 77-87 clinicians' perspectives, we aimed to obtain the perspective of physiotherapy managers on the provision of musculoskeletal physiotherapy services in primary care in Ireland. We also aimed to uncover the barriers and facilitators to musculoskeletal service delivery and to gain an insight into professional development needs among physiotherapy staff.

\section{Methods}

\section{Study design and participants}

We used a phenomenological approach to explore physiotherapy managers' views of the delivery of musculoskeletal physiotherapy services in an Irish primary care setting. The aim of a phenomenological theoretical approach is to set aside current knowledge and review concepts through the eyes of the individual experiencing them (Husserl, 1970; Lowes and Prowse, 2001). Using this approach, researchers take the view that there is no correct answer, but that each individual has a range of subjective experiences. The aim is to identify, understand, describe and maintain the subjective experiences of research participants and by doing so to develop new understanding (Husserl, 1970; Lowes and Prowse, 2001). In this study, the purpose of the interview was to enhance the researchers' understanding of the lived-in experience of managing musculoskeletal physiotherapy services from the perspective of these stakeholders. We considered each participant as an individual who interpreted the question in their own unique way (Nicholls, 2017). Semi-structured individual interviews allowed exploration of the research themes to unearth new information that was not anticipated prior to the interviews.

A list of managers $(n=36)$ was obtained from the Health Service Executive (HSE). Purposive sampling was used to ensure a geographical spread of primary care physiotherapy managers in both rural and urban settings. Potential participants were sent a participant information leaflet and asked to contact the researchers if they were interested in taking part. Written informed consent was obtained prior to conducting the interviews. The interview questions (Online Supplemental Material) were based on previous relevant literature (Minns and Bithell, 1998; Minns Lowe and Bithell, 2000), together with elements that the researchers considered pertinent. One researcher (H.F.) conducted the interviews at a 
location and time convenient to study participants. The researcher, an academic physiotherapist with a musculoskeletal clinical background, received qualitative research training and has previous qualitative research experience (French and Galvin, 2017). Each interview was audio recorded for later transcription. While several methods of determining data saturation are proposed (Fusch and Ness, 2015), we considered data saturation as achieved when the ability to obtain additional new information was attained (Guest et al., 2005). The Consolidated criteria for reporting qualitative research (COREQ) standardised reporting guidelines (Tong et al., 2007) were adhered to in the conduct and reporting of the study.

\section{Data analysis}

All interviews were transcribed by a professional transcriber. To ensure trustworthiness of the data, member checking was done with participants. Transcripts were examined using thematic analysis (Braun and Clarke, 2006), where transcripts were initially read in their entirety to get a sense of the whole conversation and, using line by line analysis, patterns and themes were identified and documented. Two researchers (R.G. and H.F.) independently coded and analysed the transcripts to identify major and minor themes using an iterative process. The suitability of the coding system was examined during this process and patterns both consistent and inconsistent with the codes were explored. The codes were designed to be understandable definitions, which could be easily interpreted and used by other independent coders. Codes were grouped into minor themes and subsequent major themes following a number of consensus meetings.

\section{Findings}

Five one-to-one interviews were held across the Republic of Ireland in two urban and three rural areas. Interviews lasted between 37 and $126 \mathrm{~min}$ (mean $=74 \mathrm{~min}, \mathrm{SD}=33 \mathrm{~min})$. Data saturation was deemed to have occurred after the fifth interview.

\section{Overview of musculoskeletal service provision in primary care}

The participating managers were responsible for between nine and 26 PCTs. A mix of self-referral based on clinical need and referral by medical card entitlement existed. All managers reported that most referrals to physiotherapy were musculoskeletal, with the GP being the primary referral source. Musculoskeletal services were based in various locations, from single rooms in small health centres to purpose-built centres with a combination of cubicles and open gym space. Two managers reported future planned physical infrastructural development. Physiotherapy appointment times varied between 30 and 45 min depending on whether a first assessment or return visit was required. Attempts to reduce treatment times to improve service efficiencies proved challenging due to the chronic and complex physical and mental health needs of many patients. Non-attendance of patients resulted in the development of strict non-attendance policies and opt-in services to optimise service efficiency across the sites. Administrative support was generally ad hoc, limited and unreliable, with physiotherapists commonly absorbing administrative duties. All managers reported that staffing levels had reduced over recent years due to economic restrictions within the HSE, predominantly due to posts being vacated when staff went on maternity leave or resigned from their posts.

\section{Qualitative themes}

Three major inter-related themes emerged from the interviews: the physiotherapist as an integral team member, optimising health service resources and barriers to health service delivery. Figure 1 displays these themes and associated subthemes.

\section{The physiotherapist an integral team member}

Within this theme, both positive and negative aspects of working in the MDT in primary care were identified. The managers perceived the physiotherapist as a central member of the PCT, with a very positive relationship with the GP:

'over about the last three to four years referrals have doubled overall, GP referrals have tripled and I'd say the bulk of that is because they're aware of primary care teams and because they've engaged somewhat with the primary care process and are aware that "oh, there's a physio for this primary care team, we'll refer there",.

Primary Health Care Research \& Development 2018; 19: 77-87 


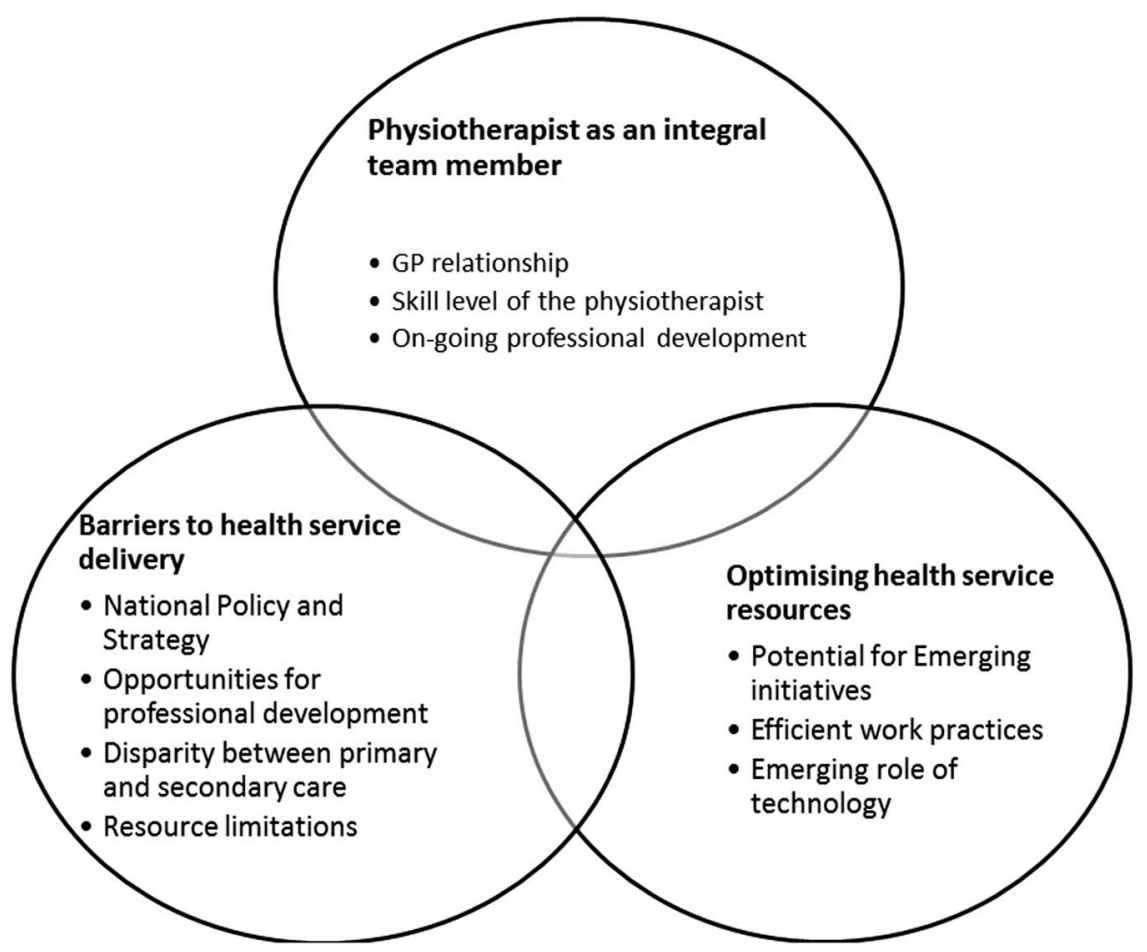

Figure 1 Overview of major and minor themes

although a full understanding of the role of physiotherapy within the PCT appeared to be a barrier:

'....the physiotherapist on the primary care team is the gateway to a range of physiotherapy services. Because GPs mightn't, they didn't know, they really had no understanding of the range of services that are being provided by acute setting and community'.

Physical location of the MDT in the same building, accommodation sharing with other MDT members and attendance at team meetings were also considered to enhance MDT working. However, while physiotherapists engaged well within the PCT, there was potential risk of isolation from their professional group.

The experience level of junior grade physiotherapists was also a subtheme. Many had completed musculoskeletal Masters degrees and were longer than three years qualified, which Primary Health Care Research \& Development 2018; 19: 77-87 is the lower cut-off for promotion to senior grade in the Irish public sector system. Although this was a positive attribute for service delivery, it was a negative factor for the therapists themselves due to limited career progression:

'They're qualified maybe six years, but I suppose that's a negative as well in that there is no prospect of a senior post coming up and they do say it to me "oh, I'm going to be a staff grade forever"'.

The challenge of being a generalist physiotherapist in primary care, treating patients across a spectrum of conditions and ages, was acknowledged as conflicting with developments towards professional body specialisation:

'The employer is looking for generalists and within the profession we've been working towards specialism. And in some ways the 
employer is not interested in any job description that has specialist in it'.

Engaging in CPD was an important role of the primary care physiotherapist, with many managers identifying work-based CPD activities such as in-service training, journal clubs and practical skills training were important to maintain musculoskeletal competency. Regular professional development planning was used to identify skills gaps and training needs. Releasing staff to attend external CPD events was identified as a challenge for all managers. All reported there was no formal HSE policy on study leave for CPD, unless undertaking Masters level education, which frequently resulted in personal financial investment by the therapists. CPD opportunities funded by the HSE were welcomed but lack of standardised policies from the HSE was frustrating for all.

'What I find with, with my staff is, that, you know, they, they spend a fortune on going to CPD. They really are, really great'.

\section{Optimising health service resources}

This theme focussed on recent initiatives to improve efficiencies in health service delivery as well as emerging opportunities for service development. Managers highlighted a number of positive elements to the evolving nature of health service delivery. These included an increase in the range of services offered to patients including gymbased exercise programmes, orthotics clinics and self-management programmes.

'for the general exercise class, we would have all age groups. And we have circuit based interval training and we find that the best way of managing very mixed case loads .... So the aim with these network services and we, is to reduce number of treatments and you know, promote self-management'.

The increased number of primary care sites was also noted as a positive development which helped to stabilise patient demand. Areas for related service development where current gaps were identified included mental health services, health promotion and women's health.
'I'm very sorry that we didn't have an option on the mental health because actually to me that's the big miss'.

Community partnerships and community engagement were highlighted as important elements of service delivery and health promotion in primary care.

'We do the move for health initiative every year and we try, you know either go out to schools or something like that. We've done large pieces around the chronic disease of trying to kind of roll it out. We've done work with the sports partnership in terms of the link between obesity and chronic disease'.

Self-referral was also perceived as a positive initiative in one centre as it served to optimise attendance and actively involve patients in selfmanagement of chronic conditions.

Staff availability, engagement and collaboration were described as key factors in optimising service delivery. The role of administrative staff was particularly valued in optimising service efficiency and effectiveness. Due to financial constraints, more efficient work practices were vital to maintain optimal patient care

'We have adapted over the last three to four years and, as I say, with twice as many referrals and virtually the same number of staff, is the equivalent of our staff being halved'.

Provision of pre-qualification student physiotherapist practice education placements served to enhance students' exposure to a continually evolving model of care as well as actively managing waiting lists:

'The third year students were assisting in group work last year, in this area and it was really positive. So as well as a learning experience, they're a tremendous resource to us. And in our health stats it showed'.

Staff rotations between acute and primary care settings served to optimise clinical exposure to Primary Health Care Research \& Development 2018; 19: 77-87 
different client groups. Junior physiotherapists were also provided with informal mentors at some sites. Invitation of guest speakers to deliver inservices and evening lectures encouraged interprofessional CPD. Joint academic and clinical partnerships served to build research links across the academic and clinical settings.

The emerging role of technology to enhance service delivery was also noted across sites, both for clinical care and as an adjunct to administrative support such as managing appointments and teleconferencing:

'One of the requests we had at the start of primary care, was to have a shared file for the MDT and it hasn't happened. And it's, it really is a very simple, I mean, the nurses now have iPhones for the infant checks on home. So I think it's starting and I do feel the technology could really assist us'.

Central appointments services were proposed to streamline services and standardise practices, but currently not in situ. Additionally, standardisation of policies around budgets and equipment procurement and allocation was noted as an area for improvement.

\section{Barriers to health service delivery}

A number of factors that impacted on service delivery and development were highlighted. Lack of national strategy around primary care service provision was perceived to result in poor standardisation of services across primary care sites:

'Well, the barriers are that the primary care teams aren't being properly managed. And the whole issue between line management, of different disciplines. And setting, you know, a lot of the initiatives in primary care team are individual led, rather than strategy led'.

Managers also highlighted the need for a national strategy around managing certain health conditions in primary care such as mental health and chronic disease. All noted limited opportunities for CPD planning and promotion within the profession.
Issues with staff retention both within physiotherapy and across the MDT were also raised:

'But I just think it's atrocious because I have to ask permission from the general manager to approve unpaid leave. So that this individual physiotherapist could spend $€ 12000$ or 16000 on the Masters and you know lose a month on unpaid leave'.

The nature and source of referrals also varied across primary care sites and inappropriate referrals were considered as a barrier to service delivery. Limited resources, including personnel, infrastructure and equipment also impacted negatively on service delivery. Reduced staffing, restricted funding and lack of auxiliary services including administrative and IT support were cited as significant barriers to optimal service delivery:

'Some primary care teams would have clerical support. I kind of, again it's at different levels, some there's kind of gold standard where clerical support acts as a receptionist physically in the building. They make appointments, take cancellations, rearrange appointments, manage the physios' diary, usually that's electronically, and maintain a database of referrals and just kind of general sort of clerical support. So that's the gold standard, that's not everywhere'.

'In the sites, we have proven that we could increase productivity by $20 \%$, if we had dedicated admin'.

Lack of cohesion both within primary care and across primary and secondary care services also impacted on service provision. Health and safety issues were highlighted as a concern, with a perceived increase in the incidence of aggression towards staff.

'Violence and aggression is a looming and large problem, employee health and safety has been largely hospital based, it's now in the last 6 or 8 months just gone out and started to take on seriously the issues in community care'. 


\section{Discussion}

The purpose of this study was to explore physiotherapy managers' views of the delivery of musculoskeletal physiotherapy services in an Irish primary care setting. Three major themes emerged from the interviews including the physiotherapist as an integral team member, optimising health service resources and barriers to health service delivery.

The managers reported that physiotherapists were considered integral team members based on anecdotal feedback. Physiotherapists have been previously identified as playing a pivotal role in PCTs by other team members, particularly in musculoskeletal health and chronic disease management, due to expertise in physical activity counselling and exercise prescription (Dufour et al., 2014). The 2017 HSE National Service Plan has identified the development of integrated care programmes for chronic disease prevention and management as a priority area in primary care (HSE, 2017). However, managers perceived there was a lack of knowledge of what the physiotherapist does which has previously been identified amongst GPs, in part due to the traditional biomedical model of patient care (Paz-Lourido and Kuisma, 2013). To this end, there is a shift internationally to increase opportunities for interprofessional education (IPE) at undergraduate level which should continue in the workplace setting (Paz-Lourido and Kuisma, 2013). While uniprofessional education remains the dominant model for delivering education for health and social care professionals, IPE is recognised as important to improve global health service delivery (World Health Organisation, 2010).

Interprofessional team working in the primary care setting is key to understanding each other's role and this can be achieved through 'top down' methods such as organisational issues related to policy, structure, space and time which can foster the 'bottom up' strategies such as the informal communication that should not be underestimated as an important method to enhance shared clinical decision making (Morgan et al., 2015).

Lack of clear HSE policy was problematic in trying to balance staff's training needs, service development requirements and service delivery. High skill levels provide the opportunity for physiotherapists to be gatekeepers in the management of musculoskeletal services in primary care (Bishop et al., 2013). Physiotherapists are specifically trained to manage symptoms and improve function and physical activity which is a key objective in the management of musculoskeletal disorders (Bishop et al., 2013). Previous research has identified that GPs lack confidence in managing such conditions (Breen et al., 2007) and direct access to physiotherapy can reduce waiting times, GP workload, improve clinical outcomes and increase patient satisfaction (Holdsworth and Webster, 2004; Ludvigsson and Enthoven, 2012; Mallett et al., 2014; Goodwin and Hendrick, 2016).

Whilst the need for specialisation with physiotherapy is considered an important focus within the profession worldwide, the need for generalist physiotherapists, akin to the GP in medicine is also recognised (Robertson et al., 2003; Bennett and Grant, 2004). There is a move towards specialisation within primary care with the development of GPs with special interests (GPwSIs) and nurse practitioner roles in primary care. In the United Kingdom, multidisciplinary musculoskeletal clinics at the primary-secondary care interface provide a 'one-stop shop' where physiotherapists and GPwSIs triage patients into appropriate management pathways (Sephton et al., 2010; Roddy et al., 2013). Such a model has the potential to improve health service delivery in Ireland with appropriate infrastructure, thereby addressing the second theme of optimising service resources. Initiatives were used to improve service efficiency, such as providing student placements. Previous research has shown that productivity does increase by taking students (Ladyshewsky, 1995; Holland, 1997). Traditionally, student physiotherapy placements were provided in secondary care in Ireland, supported by dedicated practice tutors. A recent survey has identified support for provision of placements in primary care, although clear planning and collaboration with all relevant stakeholders would be required (Reeves et al., 2013; McMahon et al., 2014). Use of information and communication technology may have the potential to increase productivity through the use of mobile phone messaging systems to increase adherence (Car et al., 2012). Technology potentially can allow patients to self-manage their own health and wellness at home (Montague, 2014). For example, smartphone apps have been effectively shown to implement behaviour change Primary Health Care Research \& Development 2018; 19: 77-87 
around physical activity (Glynn et al., 2014). Selfreferral may provide a feasible, cost-effective pathway comparable with GP referral (Mallett et al., 2014).

Barriers to service delivery included limited opportunities for capacity building and promotion both within the profession and across the MDT. While local initiatives were employed to support $\mathrm{CPD}$, the broader requirement for the development of a research culture in primary healthcare has been highlighted in several policy initiatives nationally and internationally (Farmer and Weston, 2002; Department of Health and Children, 2010; Williams et al., 2015), and evidence suggests a lack of success or skill among primary care health professionals in more advanced research activities including study design, data analysis, writing for publication and mentorship of junior colleagues/clinicians in research (Williams et al., 2015). Reduced staffing, limited funding and lack of auxiliary services were cited as significant barriers to service delivery in the current study, but these also serve as barriers to research capacity building in primary care. In an Irish context, although primary care practitioners appear to recognise the importance of research in primary care, engagement with research is poor across different professional groups. Multiple factors have been identified to explain this including a lack of research culture, absence of a supportive infrastructure including protected time, funding, research skills and supervisory support (Glynn et al., 2009).

At the time of interviewing, lack of national strategy around primary care service provision was perceived to result in a lack of standardisation of services delivery across primary care sites. Recent HSE service plans identify development of integrated clinical care pathways across acute, primary care, community and residential care settings as high priority by working with medical, nursing and therapy leads to develop and improve processes (HSE, 2017). A recent systematic review evaluating the effectiveness of integrated models of healthcare delivered at the primary-secondary interface demonstrated improvements in the management of conditions and service delivery at a modestly increased cost (Mitchell et al., 2015) but research in an Irish context would be required.

In summary, whilst three overarching themes were identified, there is much overlap across the

Primary Health Care Research \& Development 2018; 19: 77-87 subthemes. Skilled physiotherapists who can potentially improve efficiencies within the primary care system by absorbing musculoskeletal caseloads traditionally taken by GPs require on-going training and professional development, including research training. Development of more streamlined processes and use of technology can also improve work efficiency and effectiveness. Many of these changes need to be developed at national level based on national strategy with appropriate resource allocation.

\section{Implications of this research}

Some implications for both practice and research arise from this research. A greater understanding of the role of the physiotherapist within the PCT is required, both from healthcare users and other MDT members. Whilst the role of the physiotherapist as a gatekeeper for musculoskeletal conditions in secondary care is well established, the potential for such a role within primary care warrants further exploration. This could be a potentially cost-effective way of managing waiting lists and freeing up the GP's time to see patients with more complex medical needs (Ludvigsson and Enthoven, 2012; Goodwin and Hendrick, 2016). IPE should be an integral part of the healthcare undergraduate programmes and should continue through the healthcare professional's career to facilitate the development of a 'collaborative practice-ready' healthcare system (World Health Organisation, 2010). Greater involvement of physiotherapists in health policy and decision making is critical and representation of physiotherapy within the Department of Health in Ireland is crucial to facilitate strategic planning of services.

\section{Study strengths and limitations}

This is the first known study to explore the perspectives of physiotherapy managers regarding musculoskeletal physiotherapy services in primary care in Ireland and complements previous research which explored the views of senior and junior grade therapists (French and Galvin, 2017). Methodological rigour was ensured using strategies such as verification of data, participant checking, independent thematic analysis, sampling sufficiency and independent data analysis. However, we acknowledge that additional methods to ensure rigour have also been proposed including the establishment of 
intra-rater and inter-rater reliability of the codes identified (Miles and Huberman, 1994). The themes were developed only from physiotherapy managers' opinions. The views of other stakeholders such as GPs, other MDT members, HSE managers and patients should be explored to provide a more thorough understanding of the relevant issues in musculoskeletal physiotherapy in primary care.

In addition, study findings may have been influenced by the authors' research and clinical experience and should be interpreted within this context. Although a geographical representation was obtained with saturation of data, our sample of five managers could be considered small.

\section{Conclusion}

This study identified various complex inter-related factors that impact on musculoskeletal service delivery in primary care from the perspective of physiotherapy managers. These include the physiotherapist as part of the MDT, initiatives to optimise resources and barriers which impede effective service delivery. Future research should explore the views of other stakeholders to provide a more thorough understanding of the relevant issues affecting musculoskeletal service provision. Such findings can be used to inform service delivery and enhance patient care in this setting.

\section{Acknowledgements}

Emma Benton, HSE Therapy Professions Advisor who provided information regarding the structure of therapy services in primary care and the Physiotherapy managers who gave their valuable time to take part in this study.

\section{Ethical Standards}

The project received ethical approval from the Research Ethics Committee, Royal College of Surgeons in Ireland (REC-00751).

\section{Financial Support}

This work was supported by an unrestricted bursary awarded from the Irish Society of Chartered Physiotherapists on behalf of O'Driscoll O'Neil insurance.

\section{Conflicts of Interest}

None.

\section{Supplementary material}

To view supplementary material for this article, please visit https://doi.org/10.1017/S1463423617000469

\section{References}

Bennett, C.J. and Grant, M.J. 2004: Specialisation in physiotherapy: a mark of maturity. Australian Journal of Physiotherapy 50, 3-5.

Bishop, A., Foster, N.E. and Croft, P. 2013: SAPC hot topic: is it a dangerous idea to make physiotherapists the gatekeepers of frontline primary care for all patients with musculoskeletal problems? Primary Health Care Research and Development 14, 413-15.

Bornhoft, L., Larsson, M.E. and Thorn, J. 2015: Physiotherapy in Primary Care Triage - the effects on utilization of medical services at primary health care clinics by patients and sub-groups of patients with musculoskeletal disorders: a casecontrol study. Physiotherapy Theory and Practice 31, 45-52.

Braun, V. and Clarke, V. 2006: Using thematic analysis in psychology. Qualitative Research in Psychology 3, 77-101.

Breen, A., Austin, H., Campion-Smith, C., Carr, E. and Mann, E. 2007: You feel so hopeless': a qualitative study of GP management of acute back pain. European Journal of Pain 11, 21-29.

Car, J., Gurol-Urganci, I., de Jongh, T., Vodopivec-Jamsek, V., Atun, R. 2012: Mobile phone messaging reminders for attendance at healthcare appointments. Cochrane Database of Systematic Reviews 5, CD007458.

Department of Health. 2016: Department of Health statement of strategy 2016 - 2019. Retrieved 5 May 2017 from http:// www.hse.ie/eng/services/publications/serviceplans/Service-Plan2017/2017-National-Service-Plan.pdf.

Department of Health and Children. 2010: The identification of research priorities for therapy professions in Ireland. Retrieved 8 June 2016 from http://www.hrb.ie/uploads/ tx_hrbpublications/Physical_Therapies_Priorities_Main_ Report.pdf.

Desmeules, F., Roy, J.S., MacDermid, J.C., Champagne, F., Hinse, O. and Woodhouse, L.J. 2012: Advanced practice physiotherapy in patients with musculoskeletal disorders: a systematic review. BMC Musculoskeletal Disorders 13, 107.

Dufour, S.P., Lucy, S.D. and Brown, J.B. 2014: Understanding physiotherapists' roles in Ontario primary health care teams. Physiotherapy Canada 66, 234-42.

Farmer, E. and Weston, K. 2002: A conceptual model for capacity building in Australian primary health care research. Australian Family Physician 31, 1139-142.

Primary Health Care Research \& Development 2018; 19: 77-87 
French, H. and Galvin, R. 2017: Musculoskeletal services in primary care in the Republic of Ireland: an insight into the perspective of physiotherapists. Physiotherapy 103, 214-21.

Fusch, P. and Ness, L. 2015: Are we there yet? Data saturation in qualitative research. The Qualitative Report 20, 1408-416.

Glynn, L.G., Hayes, P.S., Casey, M., Glynn, F., Alvarez-Iglesias, A., Newell, J., O. Laighin, G., Heaney, D., O'Donnell, M. and Murphy, A.W. 2014: Effectiveness of a smartphone application to promote physical activity in primary care: the SMART MOVE randomised controlled trial. British Journal of General Practice 64, e384-391.

Glynn, L.G., O'Riordan, C., MacFarlane, A., Newell, J., Iglesias, A.A., Whitford, D., Cantillon, P. and Murphy, A. W. 2009: Research activity and capacity in primary healthcare: the REACH study: a survey. BMC Family Practice 10, 33.

Goodwin, R.W. and Hendrick, P.A. 2016: Physiotherapy as a first point of contact in general practice: a solution to a growing problem? Primary Health Care Research and Development 17, 89-502.

Guest, G., Bunce, A. and Johnson, L. 2005: How many interviews are enough? An experiment with data saturation and variability. Field Methods 18, 59-82.

Hay, E.M., Foster, N.E., Thomas, E., Peat, G., Phelan, M., Yates, H.E., Blenkinsopp, A. and Sim, J. 2006: Effectiveness of community physiotherapy and enhanced pharmacy review for knee pain in people aged over 55 presenting to primary care: pragmatic randomised trial. British Medical Journal 333, 995.

Health Service Executive. (HSE) 2017: HSE Service Plan 2017. Retrieved 6 May 2017 from http://www.hse.ie/eng/services/ publications/serviceplans/Service-Plan-2017/2017-NationalService-Plan.pdf.

Hill, J.C., Whitehurst, D.G., Lewis, M., Bryan, S., Dunn, K.M., Foster, N.E., Konstantinou, K., Main, C.J., Mason, E., Somerville, S., Sowden, G., Vohora, K. and Hay, E.M. 2011: Comparison of stratified primary care management for low back pain with current best practice (STarT Back): a randomised controlled trial'. Lancet 378, 1560-571.

Holdsworth, L.K. and Webster, V.S. 2004: Direct access to physiotherapy in primary care: now? - and into the future? Physiotherapy 90, 64-72.

Holland, K. 1997: Does taking students increase your waiting lists? Physiotherapy 83, 166-72.

Husserl, E. 1970. The crisis of European sciences and transcendental phenomenology. Evanston, IL: Northwestern University Press.

Jordan, K.P., Kadam, U.T., Hayward, R., Porcheret, M., Young, C. and Croft, P. 2010: Annual consultation prevalence of regional musculoskeletal problems in primary care: an observational study. BMC Musculoskeletal Disorders 11, 144.

Ladyshewsky, R.K. 1995: Enhancing service productivity in acute care inpatient settings using a collaborative clinical education model. Physical Therapy 75, 503-10.

Primary Health Care Research \& Development 2018; 19: 77-87
Lowes, L. and Prowse, M. 2001: Standing outside the interview process? The illusion of objectivity in phenomenological data generation. Journal of Nursing Studies 38, 471-80.

Ludvigsson, M.L. and Enthoven, P. 2012: Evaluation of physiotherapists as primary assessors of patients with musculoskeletal disorders seeking primary health care. Physiotherapy 98, 131-37.

MacKay, C., Canizares, M., Davis, A.M. and Badley, E.M. 2010: Health care utilization for musculoskeletal disorders. Arthritis Care Research 62, 161-69.

Mallett, R., Bakker, E. and Burton, M. 2014: Is physiotherapy self-referral with telephone triage viable, cost-effective and beneficial to musculoskeletal outpatients in a primary care setting? Musculoskeletal Care 12, 251-60.

McMahon, S., Cusack, T. and O'Donoghue, G. 2014: Barriers and facilitators to providing undergraduate physiotherapy clinical education in the primary care setting: a three-round Delphi study. Physiotherapy 100, 4-9.

Miles, M. and Huberman, A. 1994: Early steps in analysis. In Miles, M. and Huberman, A., editors Qualitative data analysis: an expanded sourcebook. Thousand Oaks, CA: Sage, pp. 64.

Minns, C. and Bithell, C. 1998: Musculoskeletal physiotherapy in GP fundholding practices. Physiotherapy 84, 84-92.

Minns Lowe, C.J. and Bithell, C.P. 2000: Musculoskeletal physiotherapy in primary care sites: survey of English NHS trusts. Physiotherapy 86, 479-85.

Mitchell, G.K., Burridge, L., Zhang, J., Donald, M., Scott, I.A., Dart, J. and Jackson, C.L. 2015: Systematic review of integrated models of health care delivered at the primarysecondary interface: how effective is it and what determines effectiveness?'. Australian Journal of Primary Health 21, 391-408.

Montague, E. 2014: The promises and challenges of health information technology in primary health care. Primary Health Care Research and Development 15, 227-30.

Morgan, S., Pullon, S. and McKinlay, E. 2015: Observation of interprofessional collaborative practice in primary care teams: an integrative literature review. International Journal of Nursing Studies 52, 1217-230.

Nicholls, D. 2017: Qualitative research. Part 2: methodologies. International Journal of Therapy and Rehabilitation 24, $71-77$.

Nordeman, L., Nilsson, B., Moller, M. and Gunnarsson, R. 2006: Early access to physical therapy treatment for subacute low back pain in primary health care: a prospective randomized clinical trial. Clinical Journal of Pain 22, 505-11.

Paz-Lourido, B. and Kuisma, R.M. 2013: General practitioners' perspectives of education and collaboration with physiotherapists in Primary Health Care: a discourse analysis. Journal of Interprofessional Care 27, 254-60.

Reeves, S., Perrier, L., Goldman, J., Freeth, D. and Zwarenstein, M. 2013: Interprofessional education: effects on professional practice and healthcare outcomes (update). Cochrane Database of Systematic Reviews 3, CD002213. 
Roberts, L. 2013: Improving quality, service delivery and patient experience in a musculoskeletal service. Manual Therapy 18, 77-82.

Robertson, V.J., Oldmeadow, L.B., Cromie, J.E. and Grant, M. J. 2003: Taking charge of change: a new career structure in physiotherapy. Australian Journal of Physiotherapy 49, 229-31.

Roddy, E., Zwierska, I., Jordan, K.P., Dawes, P., Hider, S.L., Packham, J., Stevenson, K. and Hay, E.M. 2013: Musculoskeletal clinical assessment and treatment services at the primary-secondary care interface: an observational study. British Journal of General Practice 63, e141-148.

Sephton, R., Hough, E., Roberts, S.A. and Oldham, J. 2010: Evaluation of a primary care musculoskeletal clinical assessment service: a preliminary study. Physiotherapy 96, 296-302.

Tong, A., Sainsbury, P. and Craig, J. 2007: Consolidated criteria for reporting qualitative research (COREQ): a 32-item checklist for interviews and focus groups. International Journal of Quality in Healthcare 19, 349-57.

Williams, C., Miyazaki, K., Borkowski, D., McKinstry, C., Cotchet, M. and Haines, T. 2015: Research capacity and culture of the Victorian public health allied health workforce is influenced by key research support staff and location. Australian Health Review 39, 303-11.

World Health Organisation. 2010: Framework for action on interprofessional education \& collaborative practice. Retrieved 9 June 2016 from http://www.who.int/hrh/ resources/framework_action/en/. 